\title{
Dental caries experience, oral health status and treatment needs of dental patients with autism
}

\author{
Mohamed Abdullah JABER ${ }^{1}$ \\ 1- BDS, MSc, FDSRCS, FDSRCSE, PhD, Dental School, Ajman University of Science \& Technology, (Al Jurf campus), Ajman, United Arab Emirates.
}

Corresponding address: Dr Mohamed Abdullah Jaber - Associate Professor/ Department of Surgical Sciences - College of Dentistry - Ajman University of Science \& Technology (Al Jurf campus) - Ajman, P.O. Box 346 - United Arab Emirates - Phone: (+971) 67052222 ext: 6448 - Mobile (+971) 505178052 - Fax: (+971) 67056462 - e-mail: mjaber4@hotmail.com

Received: July 16, 2009 - Modification: September 29, 2009 - Accepted: February 16, 2010

\section{ABSTRACT}

\begin{abstract}
$\mathrm{O}$ bjectives: Autism is a lifelong neurodevelopmental disorder. The aims of this study were to investigate whether children with autism have higher caries prevalence, higher periodontal problems, or more treatment needs than children of a control group of nonautistic patients, and to provide baseline data to enable comparison and future planning of dental services to autistic children. Material and Methods: 61 patients with autism aged 6-16 years ( 45 males and 16 females) attending Dubai and Sharjah Autism Centers were selected for the study. The control group consisted of 61 non-autistic patients chosen from relatives or friends of autistic patients in an attempt to have matched age, sex and socioeconomic status. Each patient received a complete oral and periodontal examination, assessment of caries prevalence, and caries severity. Other conditions assessed were dental plaque, gingivitis, restorations and treatment needs. Chi-square and Fisher's exact test of significance were used to compare groups. Results: The autism group had a male-tofemale ratio of $2.8: 1$. Compared to controls, children with autism had significantly higher decayed, missing or filled teeth than unaffected patients and significantly needed more restorative dental treatment. The restorative index (RI) and Met Need Index (MNI) for the autistic children were 0.02 and 0.3 , respectively. The majority of the autistic children either having poor $59.0 \%(36 / 61)$ or fair $37.8 \%(23 / 61)$ oral hygiene compared with healthy control subjects. Likewise, $97.0 \%(59 / 61)$ of the autistic children had gingivitis. Conclusions: Children with autism exhibited a higher caries prevalence, poor oral hygiene and extensive unmet needs for dental treatment than non-autistic healthy control group. Thus oral health program that emphasizes prevention should be considered of particular importance for children and young people with autism.
\end{abstract}

Key words: Autism. Autistic disorder. Dental caries. Dental care for disabled. Dental care for children. Oral health.

\section{INTRODUCTION}

Autism or autistic disorder is a neurodevelopment disorder characterized by impaired social interaction, communication, and restricted and repetitive behavior. These signs all begin before a child is three years old ${ }^{1}$. Autism affects many parts of the brain; how this occurs is not understood ${ }^{2}$. It is a highly variable brain development disorder ${ }^{9}$ that first appears during infancy or childhood, and generally follows a steady course without remission ${ }^{30}$. Overt symptoms gradually begin after the age of six months, become established by age two or three years ${ }^{26}$, and tend to continue through adulthood, although often in more muted form ${ }^{25}$. It is distinguished by a characteristic triad of symptoms: impairments in social interaction; impairments in communication; and restricted interests and repetitive behavior. Other aspects, such as atypical eating, are also common but are not essential for diagnosis?.

The prevalence estimated to be 1-2 per 1,000 for autism and close to 6 per 1,000 for Autism Spectrum Disorder ${ }^{20}$, with about four times as many males as females. The number of people known to have autism has increased dramatically since 
the 1980s, partly due to changes in diagnostic practice, referral patterns, availability of services, age at diagnosis, and public awareness ${ }^{8}$, though unidentified environmental risk factors cannot be ruled out ${ }^{27}$.

This complex developmental disability impairs communication, social, behavioral, and intellectual functioning. Some may express abnormal emotional and linguistic development, as well as visual and hearing impairment; others also have coexisting disabilities such as mental retardation or epilepsy, all these symptoms may complicate dental care for the affected children ${ }^{17}$. Children with autism also commonly have damaging oral habits, such as bruxism, tongue thrusting, picking at the gingiva, and lip biting.

There are few studies describing oral health and dental needs of children with autism. These studies reported no statistically significant differences in the prevalence of caries, gingivitis and degree of oral hygiene in comparison with non-autistic individuals ${ }^{6,28}$ and some studies reported a lower incidence of dental caries in autistic patients ${ }^{16,18}$.

Information on the patterns of development of the disease in the population is important because it acts as foundation for the planning of public oral health policies. Thus, the present study was carried out in an attempt to (1) describe the caries experience, treatment needs of autistic children aged 6-16 years in United Arab Emirates (UAE), and (2) provide baseline data at national and regional level to enable comparison and future planning of dental services to the autistic children.

\section{MATERIAL AND METHODS}

\section{Subjects" Selection}

Sixty-one autistic children (45 males and 16 females) attending autism centers of emirates of Dubai and Sharjah, United Arab Emirates (UAE), were selected for the study. The centers offer an intensive rehabilitation program only for children diagnosed with autism. All the children had been previously examined and diagnosed medically as autistic patients according to the center's medical records.

Consent for examining the children was obtained from the parents and respective head master.

The inclusion criteria involved were:

Diagnosis of autism;

Consent;

Age between 6 and 16 years.

Exclusion criteria were:

Dental prophylaxes in the last 6 months;

Suffering from other diseases known to influence dental caries or the severity of periodontal disease such as Down's syndrome and diabetes;
According to these criteria, 61 patients with autism were included in this study.

\section{Control group selection}

The healthy controls (61 subjects) who met the above-mentioned criteria were chosen from relatives or friends of autistic patients in an attempt to have matched age, sex, socioeconomic status and general dental care background. All controls were medically fit and none was undergoing antibiotic or antiinflammatory therapy or had undergone such therapy in the previous 6 months.

\section{Examination}

Following a complete medical history, all subjects were examined by one examiner for oral hygiene status and dental caries while seated on a dental chair using dental mirror, explorer and a periodontal probe with William's markings. The examination of the soft and hard tissues was done under flash light and regular room light. Each child accompanied by his/her teacher was brought to the examination room and is seated on an adjustable chair. "TellShow-Feel and Do" technique was used with all the children. The dmft/DMFT index was used, with codes and criteria established by the $\mathrm{WHO}^{29}$ (1997). Met Need Index (MNI), an indication of treatment received by an individual is determined using the ratio of the mean missing (M) plus filled $(F)$ teeth to mean decayed, missing and filled teeth (DMF) that is M+F/DMF. While Restorative Index (RI) which reflects the restorative care of those who have suffered the disease is measured by the ratio of filled $(F)$ to filled plus decayed teeth $(F+D)$ percent that is $F / F+D$ percent as described by Jackson ${ }^{12}$ (1973). This methodology was used systematically for all autistic and healthy control children examined. The gingival status was evaluated according to the gingival index of Loe and Silness ${ }^{15}$ (1963). Gingival status was recorded as generalized or localized gingival inflammation depending on the amount of gingival redness and bleeding during the examination. Oral hygiene was recorded as good, fair or poor according to the Simplified Oral Hygiene Index $(\mathrm{SOHI})^{10}$. Before examinations, intraobserver agreement for the diagnosis of caries was checked: one in every 10 children was re-examined to test for intraexaminer agreement. The kappa test was adopted on a tooth by tooth basis in order to check the intraexaminer agreement in caries diagnosis, in agreement with the methodology described by Peres, et al. ${ }^{21}$ (2001). Examiner agreement was high. The minimum value obtained was 0.73 , but the great majority of values were equal to one. 


\section{Data Analysis}

The data were summarized and described using relative frequencies and percentages for categorical variables (such as the presence of decayed teeth) and means for the interval scaled variables (such as the DMFT/dmft score). When the outcome variables were categorical, chi-squared and $t$-test was used to examine differences by gender, and age group for statistical significance. Data were analyzed using SPSS software, version 12.0 (SPSS Inc., Chicago, IL, USA) for Windows. Statistical significance was set at $\mathrm{P}<0.05$.

\section{RESULTS}

\section{Prevalence}

The study group comprised of 61 autistic children, 45 boys and 16 girls with a male-to-female ratio of 2.8:1 aged 6 to 16 years. The mean age of autism patients $(8.45)$ years and healthy controls (8.6 years) was very similar. Likewise, male-tofemale ratios of both groups were similar.

The overall prevalence of dental caries among autistic children was $77.0 \%(47 / 61)$ where as for the healthy controls was $46.0 \%$ (28/61) (Figure $1)$. The difference in the carious prevalence was significant $(p<0.05)$ between the two groups.

\section{Mean DMFT/dmft values}

For the children and adolescents with autism the values of the DMFT/dmft are presented in Table 1. The mean $\mathrm{dmft}$ of the autistic group was $0.80 \pm 0.2 .0$ while that of the controls was $0.30 \pm 0.3$. The mean DMFT of the autistic group was $1.6 \pm 0.64$ and $0.6 \pm 0.29$ in the controls $(p<0.05)$. There was also no significant difference between male and female. The percentage of decayed, missing and filled teeth increased with advancing age.

Met Need Index (MNI) and Restorative Index (RI) and treatment need

The mean DMFT/dmft for the 2 groups is presented (Table 2). The overall means DMFT/dmft for autistic patients was 2.4 and for healthy control was 0.9 . The treatment received and restorative care of those autistic children who have suffered the disease are presented in Table 2 . The RI for autistic group was 0.02 and MNI was 0.09 . For the control group the RI of 0.05 was found and MNI was 0.3 as 7 children had extraction done previously and 1 had amalgam restoration. As for the treatment need of the study population, the results showed that all of autistic children required oral prophylaxis, compared with $25(41 \%)$ of controls. $47(77.0 \%)$ autistic children and $28(46.0 \%)$ controls also required restorative treatment (Table 3 ).

\section{Gingival status and oral hygiene condition}

Oral hygiene and gingival status of the autistic children and healthy control subjects is shown in (Table 4). A total of $36(59.0 \%)$ individuals in the control group had good oral hygiene, compared with $2(3.3 \%)$ of the autism group. The majority of the autistic children either having poor $59.0 \%(36 / 61)$ or fair $37.8 \%(23 / 61)$ oral hygiene compared with healthy control subjects. As for the gingival status evaluation the results showed that $97.0 \%$ (59/61) of the autistic children had gingivitis which is generalized in $78.0 \%$ of the examined children or localized in $22.0 \%$ of the cases.

Table 1- Mean decayed, missing and filled teeth (dmft/ DMFT) scores of the autism group and healthy control subjects. Data are presented as mean \pm standard deviation

\begin{tabular}{lcc}
\hline & dmf & DMFT \\
\hline Group & & \\
Autistic patients & $0.8 \pm 0.20$ & $1.6 \pm 0.64$ \\
Control & $0.3 \pm 0.3$ & $0.6 \pm 0.29$ \\
$p$ & $<0.05$ & $<0.05$ \\
Gender & & \\
Autistic Female & $0.65 \pm 1.4$ & $0.50 \pm 0.49$ \\
Autistic Male & $0.75 \pm 1.5$ & $0.49 \pm 0.52$ \\
$p$ & $>0.05$ & $>0.05$ \\
\hline
\end{tabular}

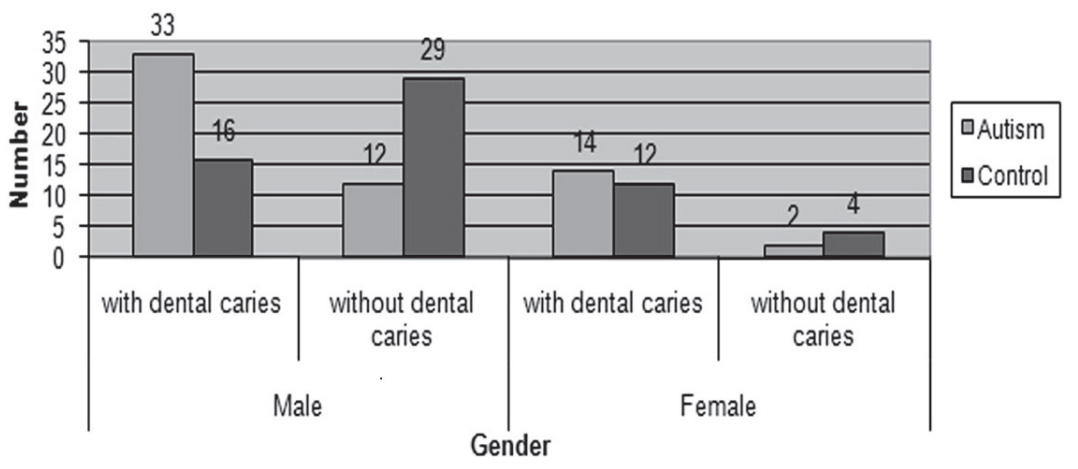

Figure 1- Distribution of the sample population according to gender and dental caries experience 
Table 2- Mean decayed, missing and filled teeth (DMFT/dmft), Restorative Index (RI) and Met Need Index (MNI) for the two groups

\begin{tabular}{ccccccccc}
\hline Age & No & D & M & F & DMFT/dmft & Mean DMFT/dmft & RI & MNI \\
\hline Autism & 47 & 105 & 8 & 3 & 116 & 2.4 & 0.02 & 0.09 \\
Control & 28 & 19 & 7 & 1 & 27 & 0.9 & 0.05 & 0.3 \\
Total & 75 & 124 & 15 & 4 & 143 & 1.9 & 0.03 & 0.13 \\
\hline
\end{tabular}

(RI) Restorative Index $=F / F+D$

(MNI) Met Need Index=M+F/DMF

Table 3- Treatment needs of the of the autism group and healthy control subjects

\begin{tabular}{lcccccc}
\hline Treatment needs & \multicolumn{2}{c}{ Autistic patients } & \multicolumn{2}{c}{ Control } & \multicolumn{2}{c}{ Total } \\
& No & $\%$ & No & $\%$ & No & $\%$ \\
\hline Oral prophylaxis & 61 & $100^{*}$ & 25 & 41.0 & 86 & 70.5 \\
Restorations & 47 & $77.0^{*}$ & 28 & 46.0 & 75 & 61.4 \\
Extractions & 3 & 5 & - & - & 3 & 2.4 \\
Endonontic treatment & 7 & 11.4 & - & - & 7 & 11.4 \\
\hline
\end{tabular}

${ }^{*} \mathrm{p}<0.05$ as compared to the control group

Table 4- Oral hygiene and gingival status of the 61 autistic patients and 61 healthy control subjects

\begin{tabular}{lcccccc}
\hline Treatment needs & \multicolumn{2}{c}{ Autistic patients } & \multicolumn{2}{c}{ Control } & \multicolumn{2}{c}{ Total } \\
& No & $\%$ & No & $\%$ & No & $\%$ \\
\hline Good oral hygiene & 2 & $3.3^{*}$ & 36 & 59.0 & 38 & 31.1 \\
\hline Fair oral hygiene & 23 & $38.0^{*}$ & 16 & 26.2 & 39 & 32.0 \\
\hline Poor oral hygiene & 36 & $59.0^{*}$ & 9 & 14.8 & 45 & 37.0 \\
\hline Gingivitis & 59 & 97.0 & 25 & 41.0 & 84 & 100 \\
Generalized & 46 & $78.0^{*}$ & 5 & 20.0 & 51 & 61.0 \\
Localized & 13 & $22.0^{*}$ & 20 & 80.0 & 33 & 39.0 \\
\hline
\end{tabular}

${ }^{*} p<0.05$ as compared to the control group

\section{DISCUSSION}

Autism, autistic disorder, childhood autism, or infantile autism are different terms given to the same condition ${ }^{2}$. The present study is the first attempt to systematically identify the problems of dental care for individuals who were diagnosed with autism in the UAE. Providing oral care to children with autism requires patience and a thorough understanding of the patient's degree of mental disability. It was reported that better understanding of the effects of autism on the behavior of an affected individual helps the dental practitioner to deliver oral health care in an empathetic and appropriate manner ${ }^{5}$.

There are no specific genetic, medical, or laboratory tests available to confirm the diagnosis of autism and the comprehensive management of autism includes parental counseling, special education in a highly structured environment, speech therapy, and social skills training, with the ultimate goal of facilitating independence in activities of daily living and self-care. Children with autism have multiple medical and behavioral problems, which make their dental treatment extremely difficult.

The studied group showed more males than females, with a ratio of $2.8: 1$, this might reflect the higher prevalence of autism in males as it was reported in other studies ${ }^{14,18}$.

The overall prevalence of dental caries among the autistic children was $77.0 \%$, the mean dmf, and DMF for all the autistic children and healthy control subjects was 2.4 and 0.9 respectively. This level of dental caries is considered low according to the WHO classification ${ }^{3}$. Comparing the results of this study with the most recent dental survey conducted in the UAE ${ }^{11}$ involving groups of healthy children with dmft of 10.2 confirmed the lower caries prevalence in the autistic children in UAE. These findings are in agreement with other regional and international studies which reported lower 
caries prevalence in autistic children ${ }^{16,18}$. The higher caries index value in permanent dentition compared with that of primary dentition is in accordance with previous studies ${ }^{18}$. However, in a recent study from Brazil Bassoukou, Nicolau and Santos ${ }^{4}$ (2009) reported that autistic individuals compared with non-autistic healthy controls, have neither a higher salivary flow rate nor a better buffer capacity of the saliva and a similar dental caries experience was observed in both primary and permanent dentitions.

The percentage of decayed, missing and filled teeth increased with advancing age. The increase attributed to the effects of time on the increased number of teeth susceptible to decay such as the second permanent molars, which erupt during adolescent period $^{19}$. In general, children with autism prefer soft and sweetened foods and they tend to pouch food inside the mouth instead of swallowing it due to poor tongue coordination, thereby increasing the susceptibility to caries ${ }^{14}$. Moreover, the risk for dental caries can be expected to be higher in these patients due to difficulties in brushing and flossing. Non-institutionalized children with autism had caries rates that were similar to the rates in functionally independent peers in a study conducted by Shapira, et al. ${ }^{28}$ (1998). Rajic and Dzingalasevic ${ }^{24}$ (1989) reported that a combined treatment, provided by a dental team and a pedopsychiatric team working together, resulted in a decreased prevalence of caries in a group of children with autism as compared to another group who did not receive any treatment. Addressing the high prevalence of dental caries among autistic children in UAE will require increased oral health promotion for parents and caregivers both at primary school age and at the preschool stage to enable them to implement effective preventive regimes for their children. Dental health education should include information/ guidance on reducing the frequency of sugary foods and drinks in the diet, good oral hygiene, use of fluoride toothpaste, and early attendance at the dentist or dental nurse for advice and care. Improving access to dental care and encouraging uptake of dental services should also form part of any health promotion strategy for autistic children.

In this study the majority of autistic children had poor oral hygiene, and almost all of them had gingivitis. These changes could be related to irregular brushing habits because of the difficulties the trainers and the parents encountered when they brushed the children's teeth. It could also be due to lack of the necessary manual dexterity of autistic children, which result in inadequate tooth brushing. Furthermore, the findings of this study reflect poor dental awareness, a lack of dental education and deficiency in receiving oral hygiene instructions from dental staff. Another possible explanation of the presence of generalized gingivitis might be the side effects of medications used to control the manifestations of autism such as psychoactive drugs or anticonvulsants, with the most common drug classes being antidepressants, stimulants, and antipsychotics ${ }^{25}$. However, Pilebro and Bäckman ${ }^{23}$ (2005) in a prospective study which included clinical examinations and structured interviews produced a series of pictures that showed a structured method and technique of tooth brushing. The pictures were placed in the bathroom or wherever tooth brushing was performed. Fourteen children with autism aged between 5 and 13 years were involved, after 12 months, the amount of visible plaque was reduced. After 18 months, most parents found maintaining good oral hygiene easier than before the study and concluded that visual pedagogy is a useful tool in helping people with autism to improve their oral hygiene.

Met Need Index (MNI) and Restorative Index (RI) of the studied autistic children were low compared with healthy control subjects. Met Need Index (MNI), an indication of treatment received by an individual is determined using the ratio of the mean missing $(M)$ plus filled $(F)$ teeth to mean decayed, missing and filled teeth (DMF) that is $M+F /$ DMF. While Restorative Index (RI) which reflects the restorative care of those who have suffered the disease is measured by the ratio of filled $(F)$ to filled plus decayed teeth $(F+D)$ percent that is $F / F+D$ percent as described by Jackson ${ }^{12}$ (1973). These results indicate the provision of dental services to this unique group of patients with high prevalence of dental caries and more treatment need compared with non-autistic healthy patients. It is therefore mean that this group of autistic children needs a lot of restorative dental care, but many of them are not receiving such treatment, as only $9.5 \%$ of them received restorative treatment compared with $29.6 \%$ of non-autistic healthy patients. This may be due to the limited access to the dental services for this group of children or due to the difficulties in controlling those children ${ }^{18}$. Consequently, only emergency treatment is sought when individual's experience dental pain and lead to filling or extraction.

The person with autism generally presents a challenge to the dentist and dental care, while behavior modification techniques have value in changing self-injurious behaviors, use of the reward system fails when the promised reward is no longer possible ${ }^{13}$. But adults with autism demonstrate increased independence by developing skills through a structured teaching ${ }^{22}$ or visual teaching ${ }^{23}$ model, such models can be used to introduce children with autism to dentistry.

Comprehensive dental treatment under general anesthesia may be required 30 percent of the time $^{14}$. In a 10 -year follow-up, patients resisted 
efforts to establish personal contact with the dental staff. Furthermore, sedative techniques were ineffective because of atypical response patterns. Use of general anesthesia was the only solution to provide necessary dental care ${ }^{25}$. When dental needs are great and attempts at behavior modification are unsuccessful, general anesthesia in the operating room creates a controlled environment where care is delivered efficiently and effectively.

In conclusion, children with autism exhibited higher caries prevalence, poor oral hygiene and extensive unmet needs for dental treatment than non-autistic healthy control group. Thus an oral health program that emphasizes prevention is considered of particular importance for children and young people with autism.

\section{ACKNOWLEDGEMENTS}

We would like to thank Dubai and Sharjah Autism Rehabilitation Center, children and their parents for giving us access to conduct this study. We also like to acknowledge the help and contribution of the doctors and other assisting staff.

\section{REFERENCES}

1- Amaral DG, Schumann CM, Nordahl CW. Neuroanatomy of autism. Trends Neurosci. 2008;31:137-45.

2- American Psychiatric Association. Pervasive developmental disorders. In: . Diagnostic and statistical manual of mental disorders. Washington DC: American Psychiatric Association; 2000. p. 69-70.

3- Barmes DE. Indicators for oral health and their implications for developing countries. Int Dent J. 1983;33:60-6.

4- Bassoukou IH, Nicolau J, Santos MT. Saliva flow rate, buffer capacity, and $\mathrm{pH}$ of autistic individuals. Clin Oral Investig. 2009;13:23-7.

5- Chew LC, King NM, O'Donnell D. Autism: the etiology, management and implications for treatment modalities from the dental perspective. Dent Update. 2006;33:70-2,74-6,78-80. 6- Fahlvik-Planefeldt C, Herrstrom P. Dental care of autistic children within the nonspecialized Public Dental Service. Swed Dent J. 2001;25:113-8.

7- Filipek PA, Accardo PJ, Baranek GT, Cook EH Jr, Dawson G, Gordon B, et al. The screening and diagnosis of autistic spectrum disorders. J Autism Dev Disord. 1999;29:439-84.

8- Fombonne E. Epidemiology of pervasive developmental disorders. Pediatr Res. 2009;65:591-8.

9- Geschwind DH. Autism: many genes, common pathways? Cell. 2008;135:391-5.

10- Greene JC, Vermillion JR. The simplified oral hygiene index. J Am Dent Assoc. 1964;68:7-13.

11- Hashim R, Thomson WM, Ayers KM, Lewsey JD, Awad M. Dental caries experience and use of dental services among preschool children in Ajman, UAE. Int J Paediatr Dent. 2006;16:257-62.
12- Jackson D. Measuring restorative dental care in communities. Br Dent J. 1973;134:385-8.

13- Johnson CD, Matt MK, Dennison D, Brown RS, Koh S. Preventing factitious gingival injury in an autistic patient. J Am Dent Assoc. 1996;127:244-7.

14- Klein U, Nowak AJ. Autistic disorder: a review for the pediatric dentist. Pediatr Dent. 1998;20:312-7.

15- Loe H, Silness J. Periodontal disease in pregnancy. I. Prevalence and severity. Acta Odontol Scand. 1963;21:533-51.

16- Loo CY, Graham RM, Hughes CV. The caries experience and behavior of dental patients with autism spectrum disorder. J Am Dent Assoc. 2008;139:1518-24.

17- Medina AC, Sogbe R, Gómez-Rey AM, Mata M. Factitial oral lesions in an autistic paediatric patient. Int J Paediatr Dent. 2003;13:130-7.

18- Murshid EZ. Oral health status, dental needs habits and behavioral attitude towards dental treatment of a group of autistic children in Riyadh, Saudi Arabia. Saudi Dent J. 2005;17:132-9.

19- National Institute of Dental and Craniofacial Research. Epidemiology and oral disease prevention program. Oral health of United State children, the national survey of dental caries in U.S. school children: 1986-1987. Bethesda: NIDCR; 1989. (NIH Publication No. 89-2247)

20- Newschaffer CJ, Croen LA, Daniels J, Giarelli E, Grether JK, Lewy SE, et al. The epidemiology of autism spectrum disorders. Annu Rev Public Health. 2007;28:235-58.

21- Peres MA, Traebert J, Marcenes W. Calibration of examiners for dental caries epidemiology studies. Cad Saúde Públ. 2001;17:1539.

22- Persson B. Brief report: a longitudinal study of quality of life and independence among adult men with autism. J Autism Dev Disord. 2000;30:61-6.

23- Pilebro C, Bäckman B. Teaching oral hygiene to children with autism. Int J Paediatr Dent. 2005;15:1-9.

24- Rajić A, Dzingalasević G. Autistic children and dental care. Acta Stomatol Croat. 1989;23:175-83.

25- Rapin I, Tuchman RF. Autism: definition, neurobiology, screening, diagnosis. Pediatr Clin North Am. 2008;55:1129-46.

26- Rogers SJ. What are infant siblings teaching us about autism in infancy? Autism Res. 2009;2:125-37.

27- Rutter $M$. Incidence of autism spectrum disorders: changes over time and their meaning. Acta Paediatr. 2005;94:2-15.

28- Shapira J, Mann J, Tamari I, Mester R, Knobler H, Yoeli Y, et al. Oral health status and dental needs of an autistic population of children and young adults. Spec Care Dentist. 1989;9:38-41. 29- World Health Organization. Oral Health Surveys. Basic method. $4^{\text {th }}$ ed. Geneva: WHO; 1997.

30- World Health Organization. Pervasive developmental disorders. In: International Statistical Classification of Diseases and Related Health Problems (ICD-10). Geneva: WHO; 2006. 